\title{
ST-Elevation Myocardial Infarction in an Adolescent
}

\author{
Priyanka Kailasha, d, Sumit Kalra $^{\mathrm{b}}$, Sameer Arora ${ }^{\mathrm{b}, \mathrm{c}}$
}

\begin{abstract}
Myocardial infarction (MI) in the elderly is often due to atherosclerotic accumulation, while an MI in younger patients is often due to embolic events. An embolic MI is rare, accounting for 2.9\% of all MIs, but has a higher risk of morbidity and mortality. The most common causes of embolic MI are infective endocarditis, atrial fibrillation, valvular heart disease, prosthetic heart valve, and mural thrombus. We present a unique case study of an ST-elevation MI (STEMI) in an adolescent, with none of the aforementioned risk factors. Our case reports a 17-year-old Caucasian male who presented to the emergency department with substernal chest pain that started while he was watching television. The pain was severe, and radiated down his left arm and up his left neck. Social history and family history were unremarkable. Vitals were within normal limits. The telemetry did not demonstrate any abnormalities. An electrocardiogram (EKG) was performed, as a rule-out, and surprisingly demonstrated an STEMI in the anterior leads with focal ST-segment depressions in the inferior leads. Cardiac catheterization was performed that demonstrated $100 \%$ stenosis in the middle to distal left anterior descending (LAD) artery. A stent was unable to be placed due to the small caliber of the occluded vessel, and medical management was started. Transesophageal echocardiogram (TEE) later demonstrated a patent foramen ovale (PFO).
\end{abstract}

Keywords: Patent foramen ovale; Myocardial infraction; Pediatric myocardial infarction; Embolic myocardial infarction

\section{Introduction}

The third universal definition defines myocardial infarction

Manuscript accepted for publication September 16, 2016

${ }^{a}$ Campbell University School of Osteopathic Medicine, 4350 US-421, Lillington, NC 27546, USA

${ }^{b}$ Department of Medicine and Cardiology, Cape Fear Medical Center, 1638 Owen Dr, Fayetteville, NC 28304, USA

'Division of Cardiology, University of North Carolina at Chapel Hill, 610 Dental Circle, Chapel Hill, NC 27599, USA

Conflict of Interest: None

${ }^{\mathrm{d} C o r r e s p o n d i n g ~ A u t h o r: ~ P r i y a n k a ~ K a i l a s h, ~ C a m p b e l l ~ U n i v e r s i t y ~ S c h o o l ~ o f ~ O s-~}$ teopathic Medicine, 4350 US-421, Lillington, NC 27546, USA.

Email: p_kailash0616@email.campbell.edu

doi: https://doi.org/10.14740/jmc2650w
(MI) as a change in troponin levels with at least one of the following: symptoms of MI, electrocardiogram (EKG) demonstrating ST changes, loss of previously viable myocardial tissue, new hypokinetic wall motion abnormalities due to ischemia or infarction, and intracoronary thrombus/emboli as seen on angiography $[1,2]$. Important diagnostic tests and tools used in diagnosing MI include performing a physical exam, obtaining cardiac biomarkers and obtaining an EKG within 10 min of patient arrival. In addition, troponin-I/troponin-T labs should be initiated on every patient in whom an MI is of suspect [2]. Chest pain is the most common presenting symptom of an MI. However, a third of the patients who are diagnosed with an MI do not present with chest pain [3]. Chest pain is often absent and/or presents atypically in populations that are older, women, and/or diabetic. Other common presenting symptoms of an MI include dyspnea, syncope, nausea, vomiting, palpitations, or cardiac arrest. Embolic MI is a very rare cause of MI, and often presents in patients with risk factors such as atrial fibrillation (AF), dilated cardiomyopathy with apical thrombus, atrial myxoma, rheumatic heart disease with mitral stenosis, and infective endocarditis.

\section{Case Report}

A 17-year-old Caucasian male with no past medical history presented to the ED with chest pain that started while watching television. The chest pain was described as substernal, severe in intensity with radiation to the left arm and neck. Social history was non-contributory. Family history was negative for premature coronary artery disease, familial hyperlipidemia, or coagulopathies. The patient was not on any medications. He denied any known drug allergies. Vital signs upon admission showed blood pressure of 130/70 $\mathrm{mm} \mathrm{Hg}$, heart rate of 70 beats per minute, respiratory rate of 20 , and oxygen saturation $100 \%$ on room air. Physical exam showed a young, white male in moderate distress who was mildly diaphoretic. The rest of his physical exam was unremarkable. Chest X-ray, CT with contrast, and an abdominal X-ray were all negative. Bedside telemetry showed sinus rhythm with no arrhythmia.

An EKG was ordered, and to the surprise of the ED physician, it demonstrated ST-segment elevations in the anterior leads along with focal ST-segment depressions in the inferior leads (Fig. 1). Initial troponin was $0.661 \mathrm{ng} / \mathrm{mL}$ (normal range: $0.000-0.045 \mathrm{ng} / \mathrm{mL}$ ). A bedside echocardiogram was performed immediately, which demonstrated mild hypokinesia of distal anterior/apical LV wall. Cardiac catheterization 


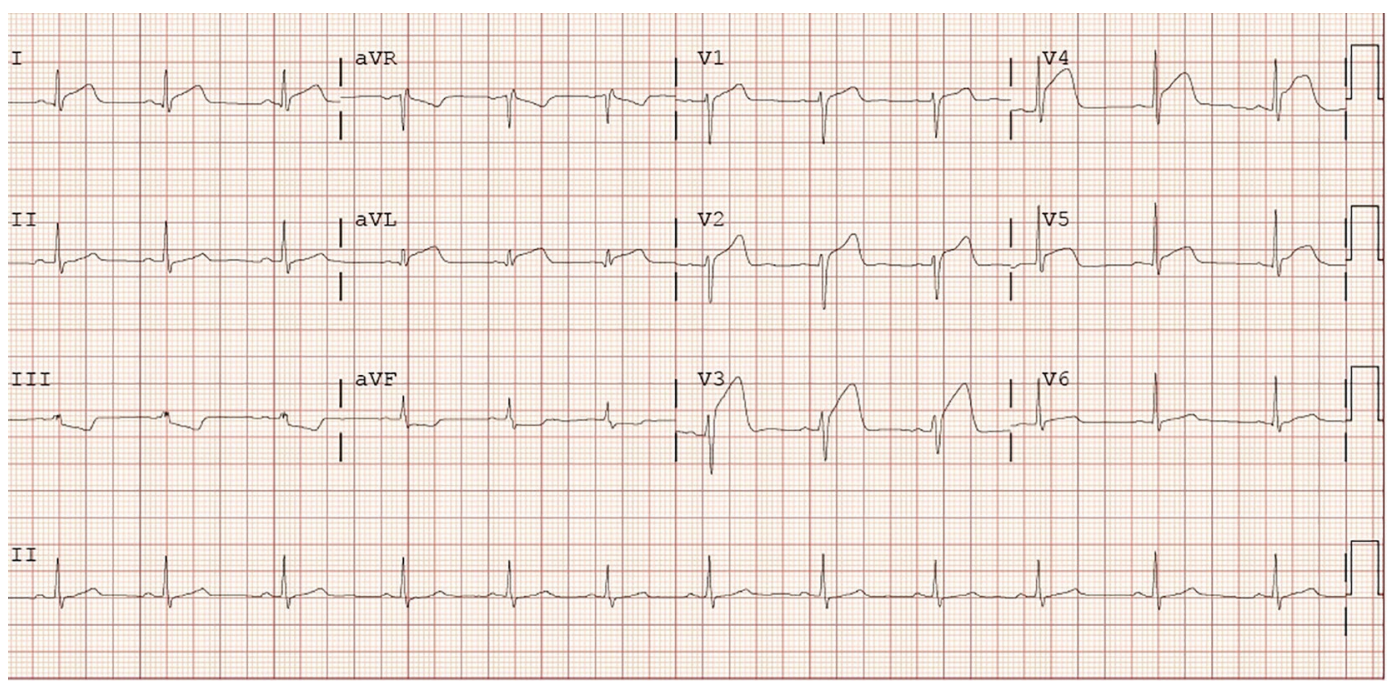

Figure 1. Electrocardiogram showing ST-segment elevations in the anterior leads along with focal ST-segment depressions in the inferior leads.

was discussed with the patient and his parents. After obtaining consent, the patient arrived in the catheterization lab and a cardiac catheterization was performed. The cardiac catheterization showed $100 \%$ stenosis in the middle to distal left anterior descending (LAD) artery (Fig. 2). Left circumflex and right coronary artery were free of disease. The size of the distal LAD was too small to intervene and hence patient was medically managed.

The patient was subsequently admitted to the cardiac inpatient service where a hematologist was consulted to rule out

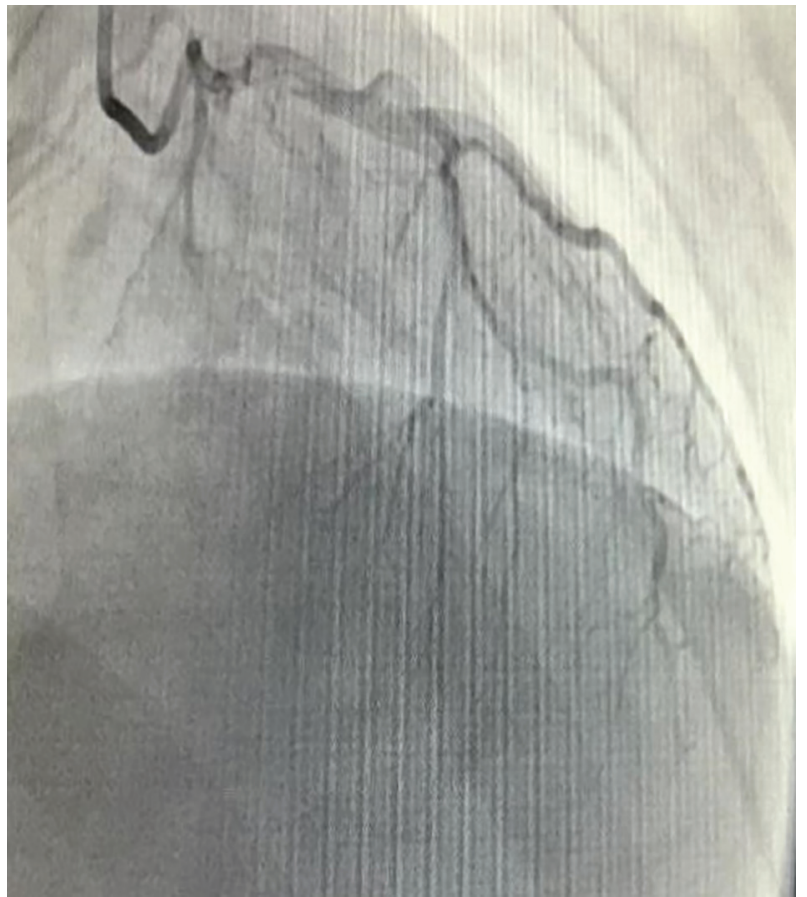

Figure 2. Cardiac catheterization demonstrating a distal left anterior descending artery occlusion. underlying thrombophilias. Cardiolipin antibodies, protein $\mathrm{C}$ and S, factor V Leiden, prothrombin mutation G20210A, and anti-thrombin deficiency were all negative [4]. Transesophageal echocardiogram (TEE) is a standard tool used for risk stratification in patients with MI. Thus, TEE is performed after optimal medical management has been achieved in this patient. The TEE confirms the presence of an intra-atrial communication, namely a patent foramen ovale (PFO) (Fig. 3a, b).

\section{Discussion}

ST-elevation MI (STEMI) is a full thickness MI that presents with elevated troponins, ST-segment elevations, and late Q wave progression on the EKG. MI due to emboli is an uncommon, but distinct clinical entity. In general, only about $4-7 \%$ of all cases of MI are due to a non-atherosclerotic etiology. Patients less than 35 years of age have a four times increased occurrence of non-atherosclerotic MIs, when compared to patients greater than 35 years of age [5].

Cardiac embolism is normally associated with an underlying condition that predisposes the patient to have an embolism. The underlying pathologies known to cause embolic MI are: $\mathrm{AF}$, tissue or mechanical valve replacement, or a large atrial septal defect (ASD). Other non-atherosclerotic causes of coronary emboli include drug abuse, coronary spasm, paradoxical embolism and rupture of sub-angiographic plaque [6].

We here present a very rare case of an anterior STEMI in a 17-year-old male with a PFO. PFO contributing to paradoxical embolism has been implicated in a cause of variety of problems such as cryptogenic stroke, peripheral artery embolism, renal and other organ infarctions [7]. PFO contributing an emboli MI is an extremely rare cause of MI. Although the instance of PFO is higher in young stroke patients, the instance of PFO in young MI patients is not higher in the control populations.

A possible hypothesis for the patient above could have 

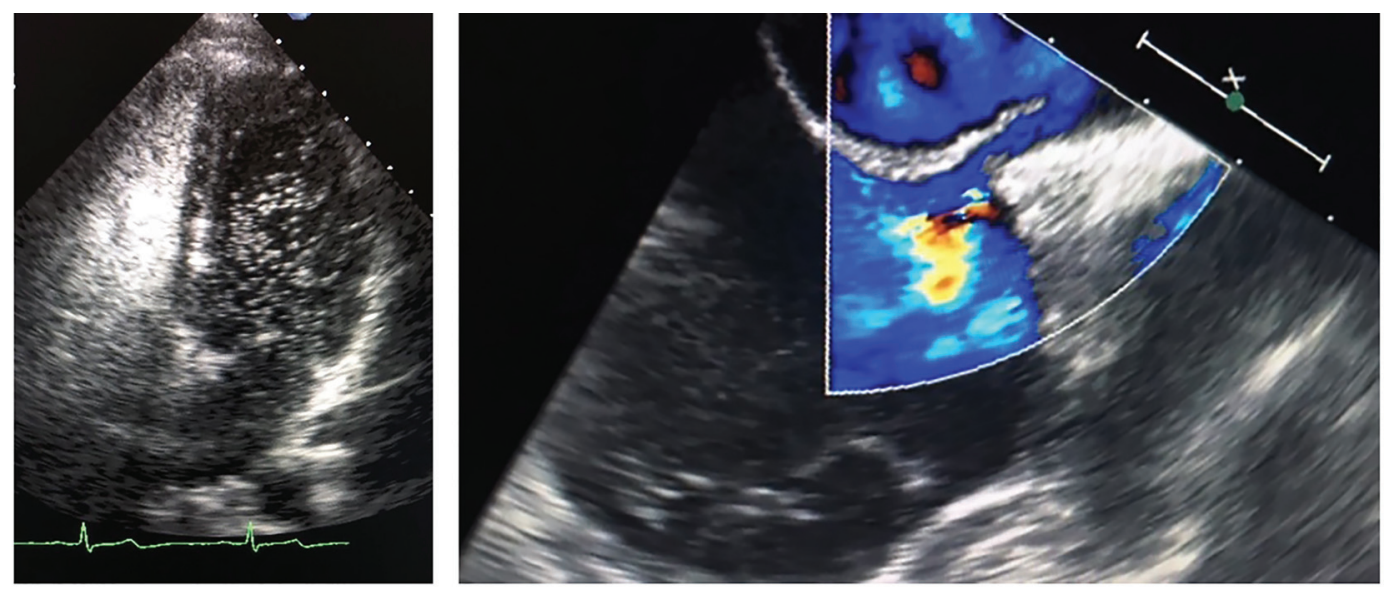

Figure 3. Transesophageal echocardiogram with (a) positive bubble study and (b) Doppler study demonstrating the interatrial communication.

been the generation of thrombus in a lower extremity vein. A small part of this thrombus embolizes into the venous circulation. This embolus travels to the heart via the inferior vena cava, and enters the right atrium. This embolus travels through the PFO, paradoxically entering the systemic circulation. The embolus is then pumped into the aorta, and travels into one of coronary arteries, in his case, the LAD. During diastole, there is a lack of perfusion to the distal LAD, thus resulting in ischemic injury, and causing an embolic MI [8].

In conclusion, patients do not always present with classic symptoms or within the expected age distributions of a disease. Although atherosclerotic is uncommon in the younger age groups, embolic MI has been reported. However, due to the devastating risk to life and limb that accompanies a missed MI, physicians need to understand unique cases such as the one above and maintain their high degree of clinical suspicion.

\section{References}

1. Charles RG, Epstein EJ. Diagnosis of coronary embolism: a review. J R Soc Med. 1983;76(10):863-869.

2. Thygesen K, Alpert JS, Jaffe AS, Simoons ML, Chait- man BR, White HD, Katus HA, et al. Third universal definition of myocardial infarction. Circulation. 2012;126(16):2020-2035.

3. Thygesen K, Alpert JS, White HD. Universal definition of myocardial infarction. Eur Heart J. 2007;28(20):25252538.

4. Bertina RM. Genetic approach to thrombophilia. Thromb Haemost. 2001;86(1):92-103.

5. Croft AP, Khan JN, Chittari MV, Varma C. Paradoxical coronary artery embolism causing acute myocardial infarction in a young woman with factor V Leiden thrombophillia. J R Coll Physicians Edinb. 2012;42(3):218220.

6. Levis JT, Schultz G, Lee PC. Acute Myocardial Infarction due to Coronary Artery Embolism in a Patient with a Tissue Aortic Valve Replacement. Perm J. 2011;15(3):82-86.

7. Cuculi F, Togni M, Meier B. Myocardial infarction due to paradoxical embolism in a patient with large atrial septal defect. J Invasive Cardiol. 2009;21(10):E184-186.

8. Siotia AK, Gunn J, Muthusamy R, Campbell S. Acute myocardial infarction in young patients: the culprit is not always a ruptured atherosclerotic plaque. Int J Clin Pract. 2007;61(9):1580-1582. 